Research Article

\title{
Folding List of Graphs Obtained from a Given Graph
}

\author{
E. M. El-Kholy $\mathbb{D}^{1}$ and H. Ahmed ${ }^{2}$ \\ ${ }^{1}$ Department of Mathematics, Faculty of Science, Tanta University, Tanta, Egypt \\ ${ }^{2}$ Department of Mathematics, Faculty of Shoubra Engineering, Banha University, Banha, Egypt \\ Correspondence should be addressed to E. M. El-Kholy; pro.entsarelkholy809@yahoo.com
}

Received 6 August 2020; Revised 26 September 2020; Accepted 27 October 2020; Published 12 December 2020

Academic Editor: Irena Lasiecka

Copyright (C) 2020 E. M. El-Kholy and H. Ahmed. This is an open access article distributed under the Creative Commons Attribution License, which permits unrestricted use, distribution, and reproduction in any medium, provided the original work is properly cited.

\begin{abstract}
In this paper, we examine the relation between graph folding of a given graph and foldings of new graphs obtained from this graph by some techniques like dual, gear, subdivision, web, crown, simplex, crossed prism, and clique-sum graphs. In each case, we obtained the necessary and sufficient conditions, if exist, for these new graphs to be folded.
\end{abstract}

\section{Introduction}

Let $G=(V, E)$ be a graph, where $V$ is the set of its vertices and $E$ is the set of its edges. By a graph, we mean a simple and finite connected graph; that is, a graph without multiple edges or loops. Let $G$ be a graph, then

(1) The dual graph $G^{*}$ of a graph $G$ is obtained by placing a vertex in every face of $G$ and an edge joining every two vertices in neighbouring faces [1].

(2) A gear graph, denoted $G_{n}$, is a graph obtained by inserting an extra vertex between each pair of adjacent vertices on the perimeter of a wheel graph $W_{n}$. Thus, $G_{n}$ has $2 n+1$ vertices and $3 n$ edges [2].

(3) If the edge $e$ joins vertices $v$ and $w$, then the subdivision of $e$ replaces $e$ by a new vertex $u$ and two new edges $v u$ and $u w$ [3]. A subdivision of a graph $G$ is a graph obtained from $G$ by applying a finite number of subdivisions of edges in succession.

(4) The web graph $W_{n, r}$ is a graph consisting of $r$ concentric copies of the cycle graphs $C_{n}$, with corresponding vertices connected by edges [4].

(5) Crown graph on $2 n$ vertices is an undirected graph with two sets of vertices $\left\{u_{1}, u_{2}, \ldots, u_{n}\right\}$ and $\left\{v_{1}, v_{2}, \ldots, v_{n}\right\}$ and with an edge from $u_{i}$ to $v_{j}$ whenever $i \neq j$ [5]. The crown graph can be viewed as a complete bipartite graph from which edges $u_{i}, v_{j}, i=1, \ldots, n$, have been removed.

(6) A simplex graph $\kappa(G)$ of an undirected graph $G$ is itself a graph, with a vertex for each clique in $G$. Two vertices of $\kappa(G)$ are joined by an edge whenever the corresponding two cliques differ in the presence or absence of a single vertex. The single vertices are called the zero vertices [6].

(7) A crossed prism graph for positive even $n$ is a graph obtained by taking two disjoint cycle graphs $C_{n}$ and adding edges $\left(v_{k}, v_{2 k+1}\right)$ and $\left(v_{k+1}, v_{2 k}\right)$ for $k=1,3, \ldots,(n-1)$ [7]. We will denote this graph by $C P_{n}$.

(8) If two graphs $G$ and $H$ each contain cliques of equal size, the clique-sum of $G$ and $H$ is formed from their disjoint union by identifying pairs of vertices in these two cliques to form a single shared clique, without deleting any of the clique edges [8].

(9) Let $G_{1}$ and $G_{2}$ be two simple graphs and $f: G_{1}$ $\longrightarrow G_{2}$ a continuous map. Then, $f$ is called a graph map, if

(i) For each vertex $v \mathcal{E} V\left(G_{1}\right), f(v)$ is a vertex in $V\left(G_{2}\right)$.

(ii) For each edge eहE $\left(G_{1}\right), f(e)$ is either a vertex or an edge of the graph $G_{2}$, i.e., $f(e) \varepsilon V\left(G_{2}\right)$ or $f(e) \varepsilon E\left(G_{2}\right)[9]$. 
(10) A graph map $f: G_{1} \longrightarrow G_{2}$ is called a graph folding if and only if $f$ maps vertices to vertices and edges to edges [10].

(11) A graph $G$ is planar if it can be drawn in the plane in such a way that no two edges meet each other except at a vertex to which they are both incident. Any such drawing is called a plane drawing of $G$. Any plane drawing of $G$ divides the plane into regions called faces.

If the edges and vertices of a face $\sigma_{i}$ of $G_{1}$ are mapped to the edges and vertices of a face $\sigma_{j}$ of $G_{2}$, then we write $f\left(\sigma_{i}\right)=\sigma_{j}$.

\section{Graph Folding of the Dual Graph}

Theorem 1. Let $G_{1}$ and $G_{2}$ be graphs and $f: G_{1} \longrightarrow G_{2} a$ graph folding. Consider the graph map $g: G_{1}^{*} \longrightarrow G_{2}^{*}$ defined by

(i) For all $v_{i}^{*} \in V\left(G_{1}^{*}\right), g\left(v_{i}^{*}\right)=v_{i}^{*}$ if and only if $f\left(\sigma_{i}\right)=\sigma_{i}$, where $\sigma_{i}$ is a face of $G_{1}$

(ii) If $f\left(\sigma_{i}\right)=\sigma_{j}$ and $f\left(\sigma_{j}\right)=\sigma_{j}$ where $\sigma_{i}$ and $\sigma_{j}$ are the neighbouring faces, then $g\left\{v_{i}^{*}, v_{j}^{*}\right\}=g\left\{v_{k}^{*}, v_{j}^{*}\right\}$ where $v_{k}^{*}$ is the vertex of the face $\sigma_{k}$ which is neighbouring to $\sigma_{j}$ but not neighbouring to $\sigma_{i}$

(iii) If $f\left(\sigma_{i}\right)=\sigma_{j}$ and $f\left(\sigma_{k}\right)=\sigma_{l}$, then $g\left(v_{i}^{*}\right)=\left(v_{i}^{*}\right)$ and $g\left(v_{k}^{*}\right)=\left(v_{j}^{*}\right)$ such that each of $\sigma_{i}, \sigma_{k}$ and $\sigma_{j}, \sigma_{l}$ are neighbouring faces

Proof. Let $f: G_{1} \longrightarrow G_{2}$ be a graph folding. Suppose that $\sigma_{i}$, $\sigma_{j}, \sigma_{k}$, and $\sigma_{l}$ are the faces of the graph $G_{1}$ such that $f\left(\sigma_{i}\right)=$ $\sigma_{j}$ and $f\left(\sigma_{j}\right)=\sigma_{j}$ where $\sigma_{i}$ and $\sigma_{j}$ are the neighbouring faces. If $\sigma_{k}$ is neighbouring to $\sigma_{j}$ and not neighbouring to $\sigma_{i}$, then there are no edges joining $v_{i}^{*}$ and $v_{k}^{*}$ in $G_{1}^{*}$, but each of $\left\{v_{i}^{*}, v_{j}^{*}\right\}$ and $\left\{v_{k}^{*}, v_{j}^{*}\right\}$ is an edge of $G_{1}^{*}$. Thus, by the given definition, $g$ maps edges to edges. Now, let $f\left(\sigma_{i}\right)=\sigma_{j}$ and $f\left(\sigma_{k}\right)=\sigma_{l}$ Then, by the given definition of $g$, it maps the vertex $v_{i}^{*}$ to $v_{l}^{*}$ and the vertex $v_{k}^{*}$ to $v_{j}^{*}$. Now, since each of the faces $\sigma_{i}, \sigma_{k}$ and $\sigma_{j}, \sigma_{l}$ are neighbouring, then each of $\left\{v_{i}^{*}, v_{k}^{*}\right\}$ and $\left\{v_{l}^{*}, v_{j}^{*}\right\}$ is an edge of $G_{1}^{*}$, i.e., the map $g$ maps edges to edges. And, consequently $g$ is a graph folding of the dual graph of $G_{1}$.

Example 1. Consider the graphs $G_{1}$ and $G_{2}$ shown in Figure 1(a). Let $f: G_{1} \longrightarrow G_{2}$ be a graph folding defined by $f\left(v_{6}\right)=\left(v_{1}\right)$ and $f\left\{\left(v_{6}, v_{2}\right), \quad\left(v_{6}, v_{3}\right),\left(v_{6}, v_{4}\right),\left(v_{6}, v_{5}\right)\right\}=$ $\left\{\left(v_{1}, v_{2}\right),\left(v_{1}, v_{3}\right),\left(v_{1}, v_{4}\right),\left(v_{1}, v_{5}\right)\right\}$, i.e., $f\left\{\sigma_{5}, \sigma_{6}, \sigma_{7}, \sigma_{8}\right\}=$ $\left\{\sigma_{1}, \sigma_{2}, \sigma_{3}, \sigma_{4}\right\}$. The graph map $g: G_{1} * \longrightarrow G_{2} *$ defined $g\left\{v_{5}^{*}, v_{6}^{*}, v_{7}^{*}, v_{8}^{*}\right\}=\left\{v_{3}^{*}, \quad v_{4}^{*}, v_{1}^{*}, v_{2}^{*}\right\}$ and $\left\{\left(v_{5}^{*}, v_{1}^{*}\right),\left(v_{5}^{*}, v_{6}^{*}\right)\right.$, $\left.\left(v_{5}^{*}, v_{7}^{*}\right),\left(v_{6}^{*}, v_{2}^{*}\right),\left(v_{6}^{*}, v_{8}^{*}\right),\left(v_{7}^{*}, v_{3}^{*}\right),\left(v_{7}^{*}, v_{8}^{*}\right),\left(v_{8}^{*}, v_{4}^{*}\right)\right\}=\left\{\left(v_{3}^{*}\right.\right.$, $\left.\left.v_{1}^{*}\right),\left(v_{3}^{*}, v_{4}^{*}\right),\left(v_{3}^{*}, v_{1}^{*}\right),\left(v_{4}^{*}, v_{2}^{*}\right),\left(v_{4}^{*}, v_{2}^{*}\right),\left(v_{1}^{*}, v_{3}^{*}\right),\left(v_{1}^{*}, v_{2}^{*}\right)\right\}$ is a graph folding, see Figure 1(b). The omitted vertices and edges, or faces, are mapped to themselves through this paper.

\section{Graph Folding of the Gear and the Subdivision Graphs}

It should be noted that any graph folding of the wheel graph $W_{n}$ maps the hup into itself.

Theorem 2. Let $W_{n}$ be a wheel graph and $G_{n}$ be the corresponding gear graph. Let $f: W_{n} \longrightarrow W_{n}$ be a graph folding. Then, the graph map $g: G_{n} \longrightarrow G_{n}$ is defined by

(i) $g\left\{v_{i}, v_{r}\right\}=\left\{v_{k}, v_{s}\right\}$ and $g\left\{v_{r}, v_{j}\right\}=\left\{v_{s}, v_{l}\right\}$ if and only if $f\left\{v_{i}, v_{j}\right\}=\left\{v_{k}, v_{l}\right\}$, where $v_{r}$ and $v_{s}$ are the extra vertices inserting between the adjacent vertices $v_{i}, v_{j}$ and $v_{k}, v_{l}$, respectively.

(ii) For the hub $v, g(v)=v$.

Proof. Let $f: W_{n} \longrightarrow W_{n}$ be a graph folding, and consider the edges $\left\{v_{i}, v_{j}\right\}, \quad\left\{v_{k}, v_{l}\right\} \in E\left(W_{n}\right)$ such that $f\left\{v_{i}, v_{j}\right\}=\left\{v_{k}, v_{l}\right\}$, i.e., $f\left\{v_{i}\right\}=\left\{v_{k}\right\}$ and $f\left\{v_{j}\right\}=\left\{v_{l}\right\}$. Now, let $v_{r}$ and $v_{s}$ be the new vertices inserted between the vertices of the edges $\left\{v_{i}, v_{j}\right\}$ and $\left\{v_{k}, v_{l}\right\}$, respectively. Then, we have four new edges $\left\{v_{i}, v_{r}\right\},\left\{v_{r}, v_{j}\right\},\left\{v_{k}, v_{s}\right\}$, and $\left\{v_{s}, v_{l}\right\} \in$ $E\left(G_{n}\right)$, but $g\left\{v_{i}, v_{r}\right\}=\left\{v_{k}, v_{s}\right\}$ and $g\left\{v_{r}, v_{j}\right\}=\left\{v_{s}, v_{l}\right\}$, i.e., the map $g$ maps edges of $G_{n}$ to other edges of $G_{n}$. Also, for all $v_{i}, v_{k} \in V\left(W_{n}\right)$ if $f\left(v_{i}\right)=v_{k}$, then $g$ maps the edge $\left\{v_{i}, v\right\}$ to the edge $\left\{v_{k}, v\right\}$ where $v$ is the hub, and consequently $g$ is a graph folding of the gear graph $G_{n}$.

Example 2. Consider the wheel graph $W_{7}$ and the corresponding gear graph $G_{7}$. Let $f: W_{7} \longrightarrow W_{7}$ be a graph folding defined by $f\left\{v_{2}, v_{3}\right\}=\left\{v_{6}, v_{5}\right\}$ and $f\left\{\left(v_{2}, v_{1}\right),\left(v_{2}\right.\right.$, $\left.\left.v_{3}\right),\left(v_{2}, v_{7}\right),\left(v_{3}, v_{4}\right),\left(v_{3}, v_{7}\right)\right\}=\left\{\left(v_{6}, v_{1}\right),\left(v_{6}, v_{5}\right),\left(v_{6}, v_{7}\right)\right.$, $\left.\left(v_{5}, v_{4}\right),\left(v_{5}, v_{7}\right)\right\}$. Then, the graph map $g: G_{7} \longrightarrow G_{7}$ defined by $g\left\{v_{8}, v_{2}, v_{9}, v_{3}, v_{10}\right\}=\left\{v_{13}, v_{6}, v_{12}, v_{5}, v_{11}\right\}$ is a graph folding, see Figure 2. In this case, $g$ maps the edges $\left(v_{1}, v_{8}\right)$, $\left(v_{8}, v_{2}\right),\left(v_{2}, v_{7}\right),\left(v_{2}, v_{9}\right),\left(v_{9}, v_{3}\right),\left(v_{3}, v_{7}\right),\left(v_{3}, v_{10}\right)$, and $\left(v_{10}\right.$, $\left.v_{4}\right)$ to the edges $\left(v_{1}, v_{13}\right),\left(v_{13}, v_{6}\right),\left(v_{6}, v_{7}\right),\left(v_{6}, v_{12}\right),\left(v_{12}, v_{5}\right)$, $\left(v_{5}, v_{7}\right),\left(v_{5}, v_{11}\right)$, and $\left(v_{11}, v_{4}\right)$, respectively.

Definition 1. For a graph $G$, if we subdivide each edge once, we get a new graph $G_{s}$, and we will call it the subdivision graph.

Theorem 3. Let $G$ be a graph and $G_{s}$ the subdivision graph of $G$. Let $f: G \longrightarrow G$ be a graph folding defined by for all $\left\{v_{i}, v_{j}\right\} \in E(G), f\left\{v_{i}, v_{j}\right\}=\left\{v_{k}, v_{l}\right\} \in E(G)$. Then, the graph map $g: G_{s} \longrightarrow G_{s}$ is defined by

(i) Mapping the edges vu and $u w$ to themselves if and only if $f$ maps the edge $v w$ to itself

(ii) $g\left\{v_{i}, u_{r}\right\}=\left\{v_{k}, u_{s}\right\}$ and $g\left\{u_{r}, v_{j}\right\}=\left\{u_{s}, v_{l}\right\}$ where $u_{r}$ and $u_{s}$ are the new vertices replaced for the edges $\left\{v_{i}, v_{j}\right\}$ and $\left\{v_{k}, v_{l}\right\}$, respectively, is a graph folding

Proof. Suppose that $f: G \longrightarrow G$ is a graph folding such that $f\left\{v_{i}, v_{j}\right\}=\left\{v_{k}, v_{l}\right\}$ where $\left\{v_{i}, v_{j}\right\}\left\{v_{k}, v_{l}\right\} \in E(G)$. Now, replace the edge $\left\{v_{i}, v_{j}\right\}$ by the new edges 

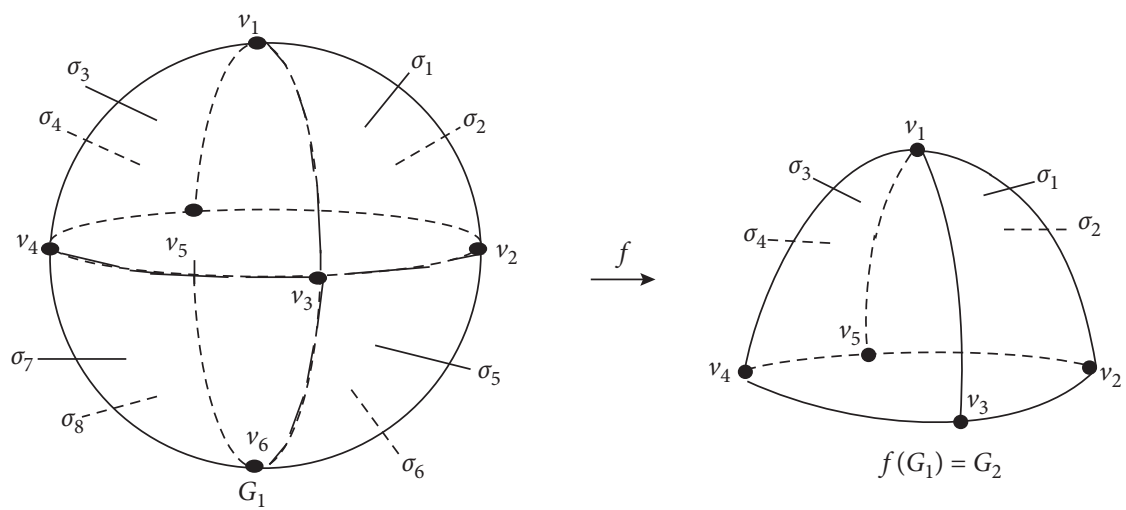

(a)
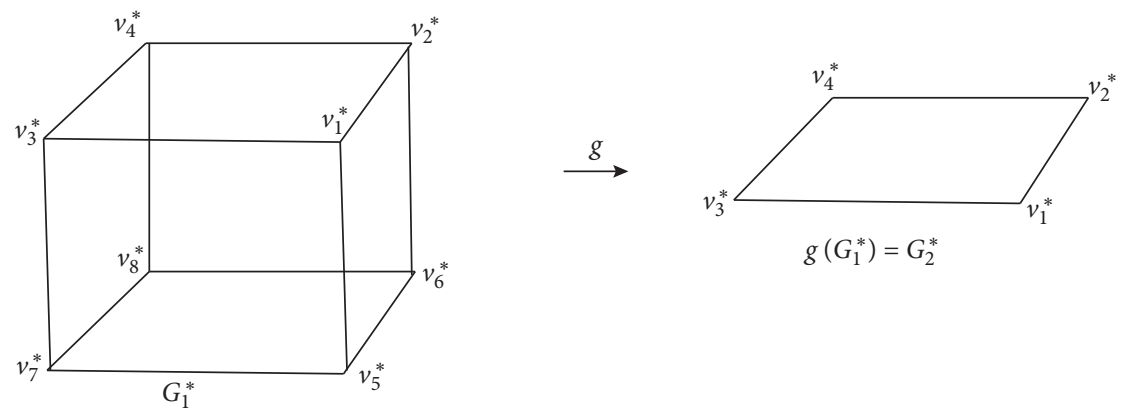

(b)

Figure 1: Graph folding of a graph and its dual graph.

$\left\{v_{i}, u_{r}\right\}\left\{u_{r}, v_{j}\right\} \in E\left(G_{s}\right)$ and the edge $\left\{v_{k}, v_{l}\right\}$ by the new edges $\left\{v_{k}, u_{s}\right\}\left\{u_{s}, v_{l}\right\} \in E\left(G_{s}\right)$. Since $g\left\{v_{i}, u_{r}\right\}=\left\{v_{k}, u_{s}\right\}$ and $g\left\{u_{r}, v_{j}\right\}=\left\{u_{s}, v_{l}\right\}$, then $g$ maps edges to edges of $G_{s}$.

Now, let $e=v w$ be an edge of $G$ such that $f(v w)=v w$. The subdivision of $e$ replaces $e$ by a new vertex $u$ and two new edges $v u$ and $u w$. Since $g(v u)=v u$ and $g(u w)=u w$, then $g$ maps edges to edges of $G_{s}$. Thus, $g$ is a graph folding.

Example 3. Consider the graph $G$ and its subdivision $G_{s}$ shown in Figure 3. Let $f: G \longrightarrow G$ be a graph folding defined by $f\left(v_{1}\right)=v_{3}$ and $f\left\{e_{1}, e_{2}\right\}=\left\{e_{4}, e_{3}\right\}$. Then, the graph map $g: G_{s} \longrightarrow G_{s}$ defined by $g\left(v_{1}, u_{1}, u_{2}\right)=$ $\left(v_{3}, u_{4}, u_{3}\right)$ and $g\left\{e_{1}^{\prime}, e_{2}^{\prime}, e_{7}^{\prime}, e_{8}^{\prime}\right\}=\left\{e_{4}^{\prime}, e_{3}^{\prime}, e_{6}^{\prime}, e_{5}^{\prime}\right\}$ is a graph folding.

\section{Graph Folding of the Web and Crown Graphs}

Theorem 4. Let $C_{n}$ be a cycle graph, where $V\left(C_{n}\right)=$ $\left\{v_{1}, \ldots, v_{n}\right\}, n$ is even, and $W_{n, r}$ be the web graph where $V\left(W_{n, r}\right)=\left\{v_{1}, \ldots, v_{n}, v_{n+1}, \ldots, v_{2 n}, v_{2 n+1}, \ldots, v_{3 n}, \ldots\right.$, $\left.v_{(r-1) n+1}, \ldots, v_{r n}\right\}$. Let $f: C_{n} \longrightarrow C_{n}$ be a graph folding defined by for all $v_{i} \in V\left(C_{n}\right), f\left(v_{i}\right)=v_{j} \in V\left(C_{n}\right)$. Then, the graph map $g: W_{n, r} \longrightarrow W_{n, r}$ is defined by

(i) For all $v_{i} \in V\left(C_{n}\right), g\left(v_{i}\right)=f\left(v_{i}\right)=v_{j}$

(ii) For all $v_{i+(s-1) n} \in V\left(W_{n, r}\right), \quad s=2, \ldots, r$, then $g\left(v_{i+(s-1) n}\right)=v_{j+(s-1) n}$
Proof. Let $C_{n}$ be a cycle graph with even vertices and $f: C_{n} \longrightarrow C_{n}$ a graph folding defined by for all $v_{i} \in V\left(C_{n}\right), f\left(v_{i}\right)=v_{j} \in V\left(C_{n}\right)$. Consider the vertices $v_{i}, v_{j}, v_{k}, v_{l} \in V\left(C_{n}\right)$ such that $f$ maps the edge $\left\{v_{i}, v_{k}\right\}$ to the edge $\left\{v_{j}, v_{l}\right\}$. For the vertices $v_{i} \in V\left(C_{n}\right), g\left(v_{i}\right)=f\left(v_{i}\right)=v_{j}$, and hence $g$ is a graph folding. If $s=2$, then $g\left\{v_{i}, v_{i+n}\right\}=\left\{v_{j}, v_{j+n}\right\}$ and $g\left\{v_{i+n}, v_{k+n}\right\}=\left\{v_{j+n}, v_{l+n}\right\}$, i.e., $g$ maps edges to edges. The same procedure can be done if $s=3,4, \ldots, r$. Thus, $g$ is a graph folding. For illustration, see Figure 4.

Example 4. Consider the cycle graph $C_{4}$. Let $f: C_{4} \longrightarrow C_{4}$ be a graph folding defined by $f\left(v_{2}\right)=\left(v_{4}\right)$ and $f\left\{\left(v_{2}, v_{1}\right),\left(v_{2}, v_{3}\right)\right\}=\left\{\left(v_{4}, v_{1}\right),\left(v_{4}, v_{3}\right)\right\}$. The graph map $g: W_{4,3} \longrightarrow W_{4,3}$ defined by $g\left\{v_{2}, v_{6}, v_{10}\right\}=\left\{v_{4}, v_{8}, v_{12}\right\}$ and $g\left\{\left(v_{2}, v_{1}\right),\left(v_{2}, v_{3}\right),\left(v_{2}, v_{6}\right),\left(v_{6}, v_{5}\right),\left(v_{6}, v_{7}\right),\left(v_{6}, v_{10}\right),\left(v_{10}, v_{9}\right)\right.$, $\left.\left(v_{10}, v_{11}\right)\right\}=\left\{\left(v_{4}, v_{1}\right),\left(v_{4}, v_{3}\right),\left(v_{4}, v_{8}\right),\left(v_{8}, v_{5}\right),\left(v_{8}, v_{7}\right),\left(v_{8}\right.\right.$, $\left.\left.v_{12}\right),\left(v_{12}, v_{9}\right),\left(v_{12}, v_{11}\right)\right\}$ is a graph folding, see Figure 5.

Theorem 5. Any crown graph $G$ of $2 n$ vertices can be folded to an edge.

Proof. Let $G$ be a crown graph on $2 n$ vertices, and let $A=\left\{u_{1}, u_{2}, \ldots, u_{n}\right\}$ and $B=\left\{v_{1}, v_{2}, \ldots, v_{n}\right\}$ be the two sets of vertices of $G$. Now, a graph folding $f: G \longrightarrow G$ can be defined by mapping all the vertices of $A$ to one vertex of $A$, say $u_{i}$, and all the vertices of $B$ to one vertex of $B$, say $v_{j}$. Thus, the image $f(G)$ is the edge $\left\{u_{i}, v_{j}\right\} \in E(G)$. Thus, $f$ is a graph folding. For illustration, see Figure 6. 


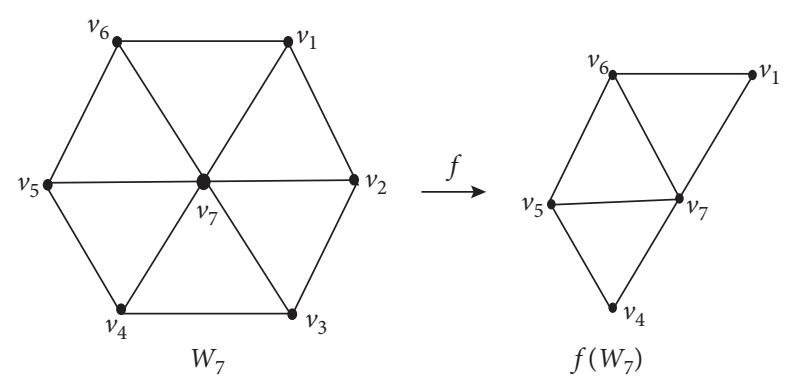

(a)

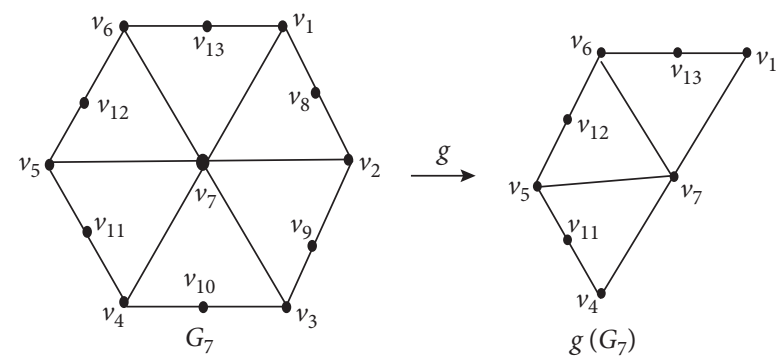

(b)

FIgURE 2: Graph folding of the wheel graph $W_{7}$ and the gear graph $G_{7}$.

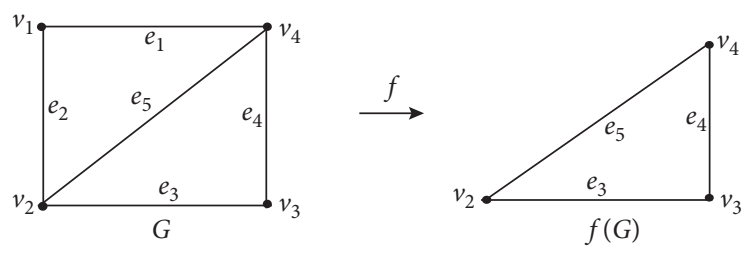

(a)

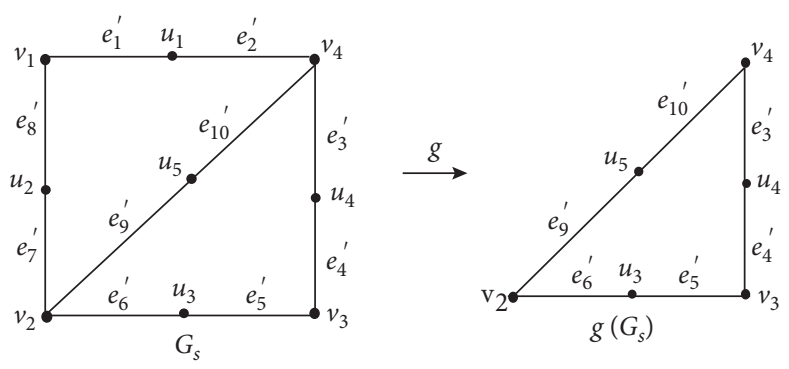

(b)

FIGURE 3: Graph folding of a graph and its subdivision graph.

\section{Graph Folding of the Simplex and Crossed Prism Graphs}

Theorem 6. Let $G$ be a graph and $f: G \longrightarrow G$ a graph folding. Then, the graph map $g: k(G) \longrightarrow k(G)$ is defined by

(i) For a zero vertex $v \in V(k(G))$, if $f\left(v_{i}\right)=v_{k}$, then $g\left\{v_{i}, v\right\}=\left\{v_{k}, v\right\}$ where $v_{i}, v_{k} \in V(G)$.

(ii) If $\left\{v_{i}, v_{j}\right\}$ and $\left\{v_{k}, v_{l}\right\}$ are cliques of $G$ such that $f\left\{v_{i}, v_{j}\right\}=\left\{v_{k}, v_{l}\right\}$, then $g\left\{v_{i}, v_{r}\right\}=\left\{v_{k}, v_{s}\right\}$ and $g\left\{v_{r}, v_{j}\right\}=\left\{v_{s}, v_{l}\right\}$ where $v_{r}$ and $v_{s}$ are the new vertices of the cliques $\left\{v_{i}, v_{j}\right\}$ and $\left\{v_{k}, v_{l}\right\}$, respectively.

(iii) If $\sigma=\left\{v_{i}, v_{j}, v_{k}\right\}$ and $\delta=\left\{v_{l}, v_{m}, v_{n}\right\}$ are cliques of $G$ such that $f\left\{v_{i}, v_{j}, v_{k}\right\}=\left\{v_{l}, v_{m}, v_{n}\right\}$, then $g\left\{u, v_{r_{\mu}}\right\}$ $=\left\{w, v_{s_{\mu}}\right\}, \mu=1,2,3$, where $u$ and $w$ are the new vertices of the two cliques $\sigma$ and $\delta$ and $v_{r_{\mu}}$ and $v_{s_{\mu}}$ are the new vertices of the edges of $\sigma$ and $\delta$, respectively.

And so on. 


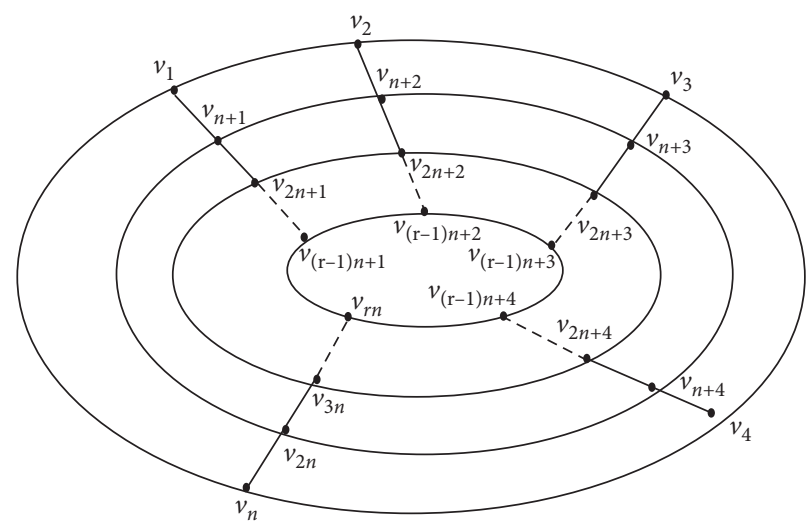

Figure 4: The web graph.

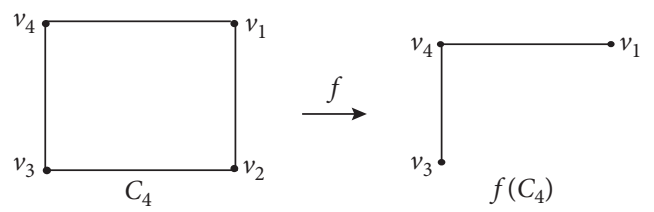

(a)

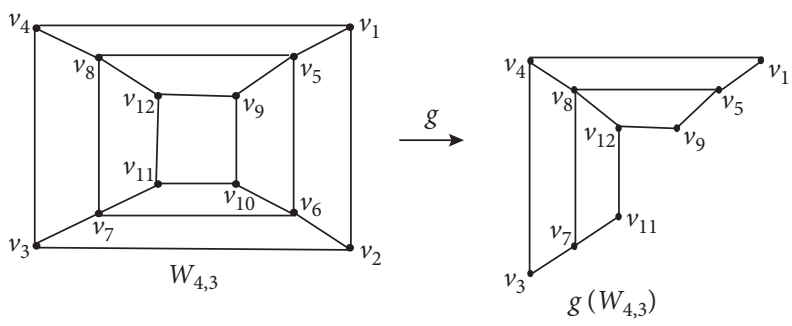

(b)

FIgURE 5: Graph folding of the cycle graph $C_{4}$ and the web graph $W_{4,3}$.

Proof. Let $G$ be a graph and $f: G \longrightarrow G$ a graph folding

(i) Consider the vertices $v_{i}, \quad v_{k} \in V(G)$ such that $f\left(v_{i}\right)=v_{k}$. Let $v$ be a zero vertex of $V(k(G))$, and then by the given definition of $g$, it maps the vertex $v$ onto itself. Then, we get new edges $\left\{v_{i}, v\right\},\left\{v_{k}, v\right\} \in E(k(G))$ but $g\left\{v_{i}, v\right\}=\left\{v_{k}, v\right\}$, i.e., $g$ maps edges to edges of $(k(G))$.

(ii) Consider the cliques $\left\{v_{i}, v_{j}\right\}$ and $\left\{v_{k}, v_{l}\right\}$ of $G$ such that $f\left\{v_{i}, v_{j}\right\}=\left\{v_{k}, v_{l}\right\}$. Let $v_{r}$ and $v_{s}$ be the new vertices of the two cliques, respectively; then we have new four edges $\left\{v_{i}, v_{r}\right\},\left\{v_{r}, v_{j}\right\},\left\{v_{k}, v_{s}\right\}$, and $\left\{v_{s}, v_{l}\right\} \in E(k(G)) \quad$ but $g\left\{v_{i}, v_{r}\right\}=\left\{v_{k}, v_{s}\right\} \quad$ and $g\left\{v_{r}, v_{j}\right\}=\left\{v_{s}, v_{l}\right\}$, i.e., $g$ maps edges to edges.

(iii) Finally, let $\sigma=\left\{v_{i}, v_{j}, v_{k}\right\}$ and $\delta=\left\{v_{l}, v_{m}, v_{n}\right\}$ be cliques of $G$ such that $f\left\{v_{i}, v_{j}, v_{k}\right\}=\left\{v_{l}, v_{m}, v_{n}\right\}$. And, considering the new vertices $u$ and $w$ of the two cliques $\sigma$ and $\delta$, respectively. Then, we have new edges $\left\{u, v_{r_{\mu}}\right\}\left\{w, v_{s_{\mu}}\right\} \in E(k(G)) \mu=1,2,3$, where $v_{r_{\mu}}$ and $v_{s_{\mu}}$ are the new vertices of the edges of the cliques $\sigma$ and $\delta$, respectively. Then, the map $g$ maps the new edges of the boundary of $\sigma$ to the new edges of the boundary of $\delta$ according to the rule (ii), and $g\left\{u, v_{r_{\mu}}\right\}=\left\{w, v_{s_{\mu}}\right\}$ where $\left\{u, v_{r_{\mu}}\right\}\left\{w, v_{s_{\mu}}\right\} \in$ $E(k(G))$. For illustration, see Figure 7. Hence, $g$ is a graph folding of the simplex graph $k(G)$.

Example 5. Let $G$ be the graph shown in Figure $8(\mathrm{a})$ and $f: G \longrightarrow G$ the graph folding defined by $f\left\{v_{3}, v_{6}, v_{8}\right\}=$ $\left\{v_{1}, v_{4}, v_{7}\right\}$ and $f\left\{\left(v_{5}, v_{6}\right),\left(v_{2}, v_{3}\right),\left(v_{3}, v_{6}\right),\left(v_{6}, v_{8}\right),\left(v_{3}\right.\right.$, $\left.\left.v_{8}\right)\right\}=\left\{\left(v_{5}, v_{4}\right),\left(v_{2}, v_{1}\right),\left(v_{1}, v_{4}\right),\left(v_{4}, v_{7}\right),\left(v_{1}, v_{7}\right)\right\}$. Then, the graph map $g: k(G) \longrightarrow k(G)$ defined by $g\left\{v_{3}, v_{6}, v_{8}\right.$, $\left.u_{7}, u_{8}, u_{9}, u_{10}, u_{11}, w_{2}\right\}=\left\{v_{1}, v_{4}, v_{7}, u_{4}, u_{5}, u_{3}, u_{2}, u_{1}, w_{1}\right\}$ and $g\left\{\left(v_{3}, V\right),\left(v_{6}, V\right), \quad\left(v_{8}, V\right),\left(v_{5}, u_{8}\right), \quad\left(u_{8}, v_{6}\right),\left(v_{2}, u_{7}\right),\left(u_{7}\right.\right.$, $\left.v_{3}\right),\left(v_{3}, u_{9}\right),\left(u_{9}, v_{6}\right), \quad\left(v_{6}, u_{10}\right),\left(u_{10}, v_{8}\right),\left(v_{3}, u_{11}\right),\left(u_{11}, v_{8}\right)$, $\left.\left(w_{2}, u_{9}\right),\left(w_{2}, u_{10}\right), \quad\left(w_{2}, u_{11}\right)\right\}=\left\{\left(v_{1}, V\right), \quad\left(v_{4}, V\right),\left(v_{7}, V\right)\right.$, $\left(v_{5}, u_{5}\right),\left(u_{5}, v_{4}\right),\left(v_{2}, u_{4}\right),\left(u_{4}, v_{1}\right),\left(v_{1}, u_{3}\right),\left(u_{3}, v_{4}\right),\left(v_{4}, u_{2}\right)$, $\left.\left(u_{2}, v_{7}\right),\left(v_{1}, u_{1}\right),\left(u_{1}, v_{7}\right),\left(w_{1}, u_{3}\right),\left(w_{1}, u_{2}\right),\left(w_{1}, u_{1}\right)\right\}$ is a graph folding, see Figure 8(b).

Theorem 7. Let $C_{n}$ be a cycle graph of even vertices and $f: C_{n} \longrightarrow C_{n}$ a graph folding. Consider the graph map $g: C P_{n} \longrightarrow C P_{n}$ such that for all $e \in E\left(C_{n}\right), g\{e\}=f\{e\}:$ 


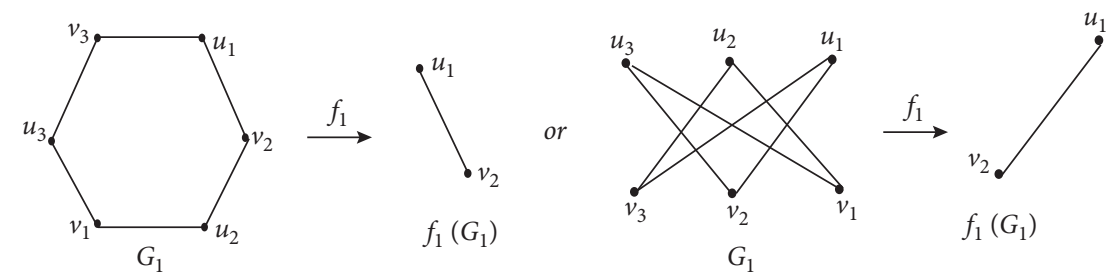

(a)
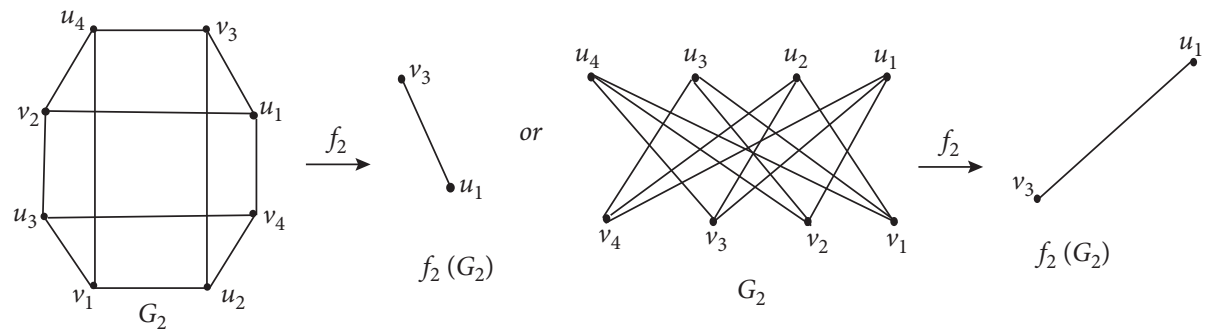

(b)

FIGURE 6: Folding crown graphs with six and eight vertices to an edge. (a) $f_{1}=\left\{u_{1}, u_{2}, u_{3}\right\}=u_{1}, f_{1}=\left\{v_{1}, v_{2}, v_{3}\right\}=v_{2}$ and (b) $f_{2}=\left\{u_{1}, u_{2}, u_{3}, u_{4}\right\}=u_{1}, f_{2}=\left\{v_{1}, v_{2}, v_{3}, v_{4}\right\}=v_{3}$.
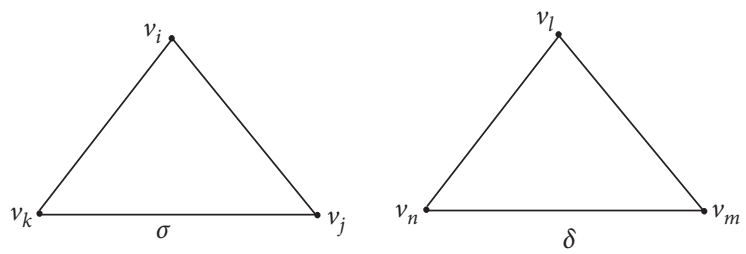

(a)
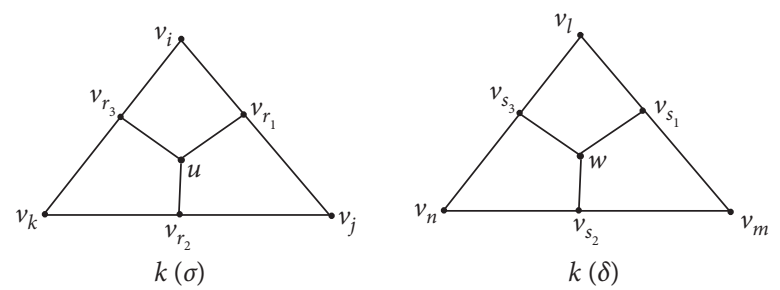

(b)

Figure 7: The simplex graph.

(i) If $g\left\{v_{2 k}, v_{2 k+1}\right\}=\left\{v_{2 k+i}, v_{2 k+i+1}\right\}$ whenever $f\left\{v_{k}, v_{k+1}\right\}$

$=\left\{v_{k+i}, v_{k+i+1}\right\}$, then $g$ is a graph folding

(ii) If $g\left\{v_{2 k}, v_{2 k+1}\right\}=\left\{v_{2 k+i}, v_{2 k+1}\right\}$ whenever $f\left\{v_{k}, v_{k+1}\right\}$ $=\left\{v_{k+i}, v_{k+1}\right\}$, then $g$ is not a graph folding

Proof (i) Let $f: C_{n} \longrightarrow C_{n}$ be a graph folding, and consider the edges $\left\{v_{k}, v_{k+1}\right\}\left\{v_{k+i}, v_{k+i+1}\right\} \in E\left(C_{n}\right)$ such that $f\left\{v_{k}, v_{k+1}\right\}=\left\{v_{k+i}, v_{k+i+1}\right\}$. Now, $g\{e\}=f\{e\}$ for all $e \in E\left(C_{n}\right)$. Also, $g\left\{v_{2 k}, v_{2 k+1}\right\}=\left\{v_{2 k+i}, v_{2 k+i+1}\right\}$, i.e., $g$ maps the edge $\left\{v_{2 k}, v_{2 k+1}\right\} \in E\left(C P_{n}\right)$ to the edge $\left\{v_{2 k+i}, v_{2 k+i+1}\right\} \in E\left(C P_{n}\right)$. Consider one of the new edges of $C P_{n}$, e.g., $\left\{v_{k+1}, v_{2 k}\right\}$. Then, $g\left\{v_{k+1}, v_{2 k}\right\}=\left\{v_{k+i+1}, v_{2 k+i}\right\} \in E\left(C P_{n}\right)$, i.e., $g$ maps edges to edges, and hence $g$ is a graph folding.

(ii) Now, let $f\left\{v_{k}, v_{k+1}\right\}=\left\{v_{k+i}, v_{k+1}\right\}$, and we know that for all $e \in E\left(C_{n}\right), g\{e\}=f\{e\}$. Also, for the edge $\left\{v_{2 k}, v_{2 k+1}\right\} \in E\left(C P_{n}\right) g\left\{v_{2 k}, v_{2 k+1}\right\}=\left\{v_{2 k+i}, v_{2 k+1}\right\}$ $\in E\left(C P_{n}\right)$, but if we consider one of the new edges of $C P_{n}$, e.g., $\left\{v_{k}, v_{2 k+1}\right\}$, then $g\left\{v_{k}, v_{2 k+1}\right\}=\left\{v_{k+i}, v_{2 k+1}\right\}$ which is not an edge of $C P_{n}$, and hence $g$ is not a graph folding.

Example 6. Consider the cycle graph $C_{4}$, and let $f_{1}: C_{4} \longrightarrow C_{4}$ be a graph folding defined by $f_{1}\left(v_{1}\right)=\left(v_{3}\right)$ 

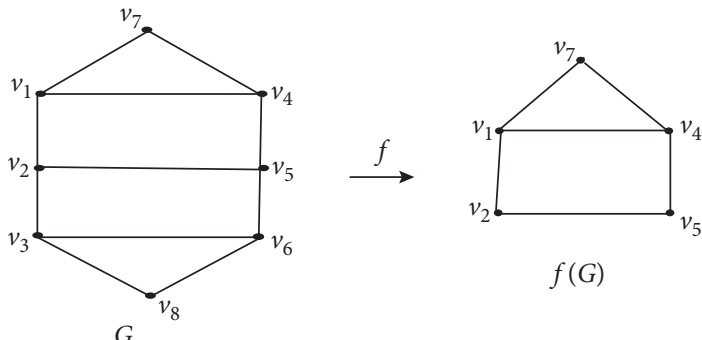

$f(G)$

(a)
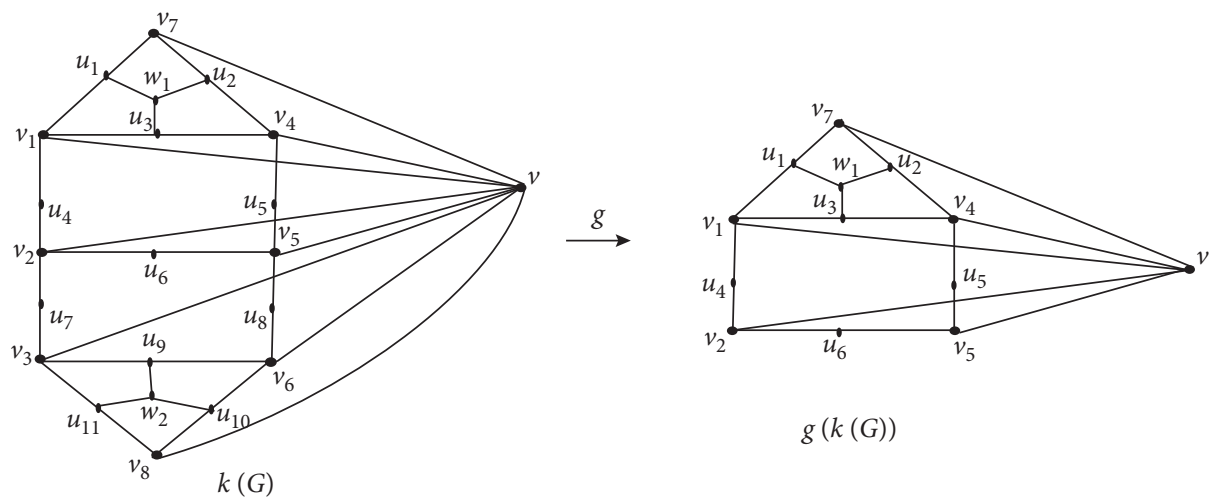

$g(k(G))$

(b)

Figure 8: Graph folding of the simplex graph.
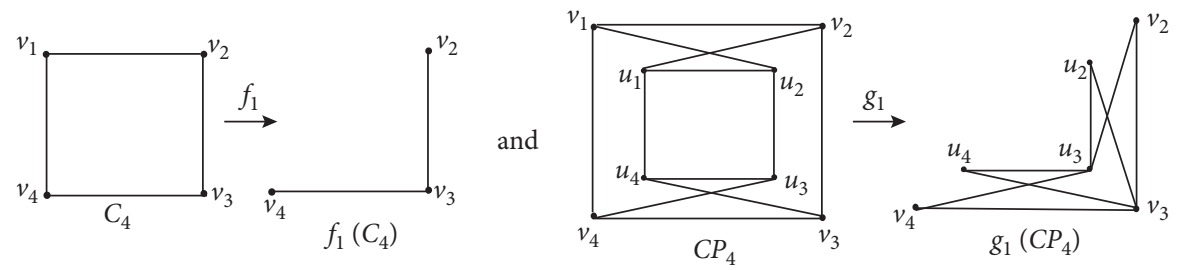

(a)
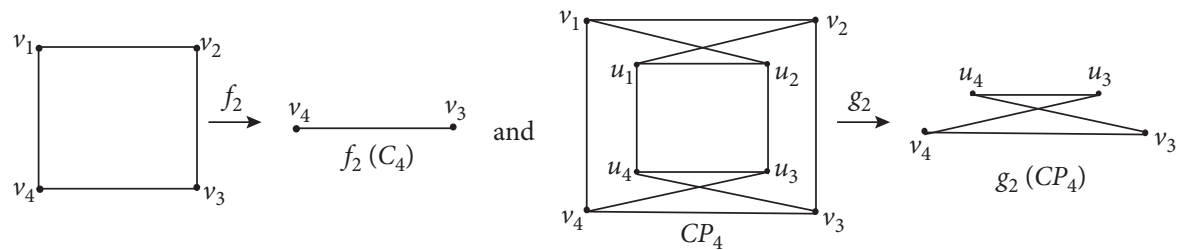

(b)

FIgURE 9: The crossed prism graph may or may not be folded.

and $f_{1}\left\{\left(v_{1}, v_{2}\right),\left(v_{1}, v_{4}\right)\right\}=\left\{\left(v_{3}, v_{2}\right)\left(v_{3}, v_{4}\right)\right\}$. The graph map $g_{1}: C P_{4} \longrightarrow C P_{4}$ defined by $g_{1}\left\{v_{1}, u_{1}\right\}=\left\{v_{3}, u_{3}\right\}$ is not a graph folding since $g_{1}\left(v_{1}, u_{2}\right)=\left(v_{3}, u_{2}\right) \notin E\left(C P_{4}\right)$, see Figure 9(a). While if $f_{2}: C_{4} \longrightarrow C_{4}$ is a graph folding defined by $f_{2}\left(v_{1}, v_{2}\right)=\left(v_{3}, v_{4}\right) \quad$ and $f_{2}\left\{\left(v_{1}, v_{2}\right),\left(v_{1}, v_{4}\right)\right.$, $\left.\left(v_{2}, v_{3}\right)\right\}=\left\{\left(v_{3}, v_{4}\right),\left(v_{3}, v_{4}\right),\left(v_{4}, v_{3}\right)\right\}$, then the graph map $g_{2}: C P_{4} \longrightarrow C P_{4}$ defined by $g_{2}\left\{v_{1}, v_{2}, u_{1}, u_{2}\right\}=\left\{v_{3}, v_{4}\right.$, $\left.u_{3}, u_{4}\right\}$ and $g_{2}\left\{\left(v_{1}, v_{2}\right),\left(v_{1}, v_{4}\right),\left(v_{2}, v_{3}\right),\left(u_{1}, u_{2}\right),\left(u_{1}, u_{4}\right)\right.$, 

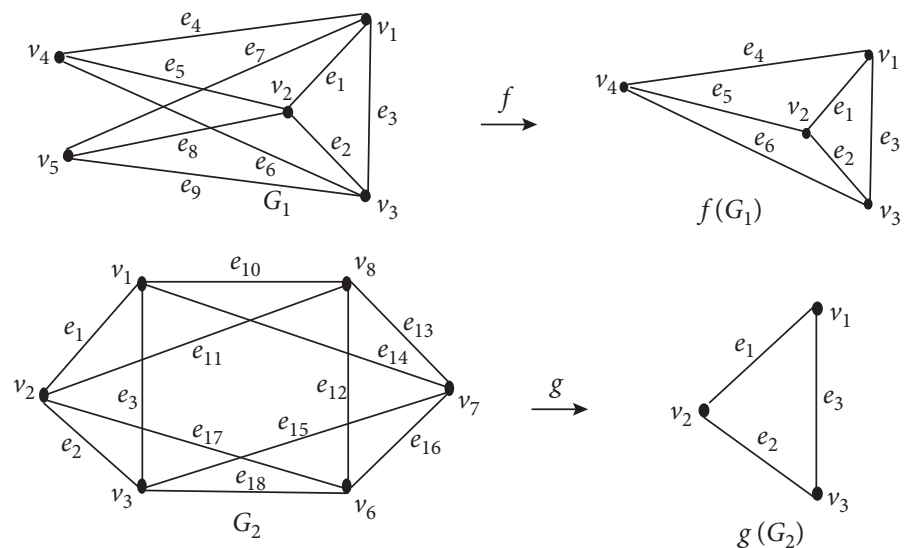

(a)

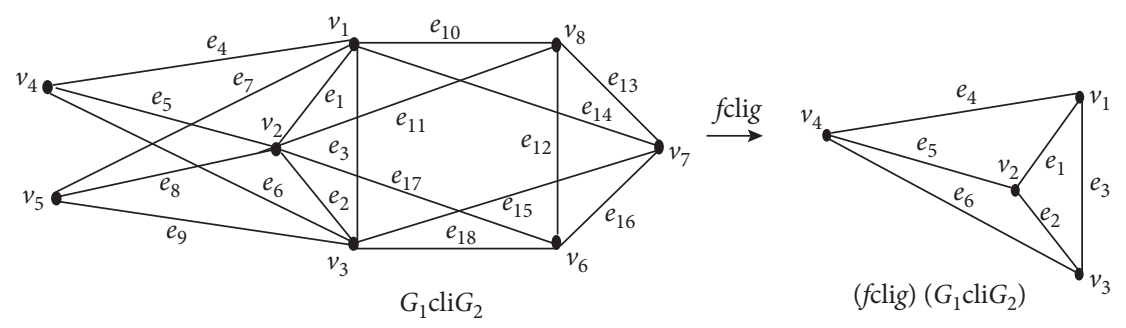

(b)

FIGURE 10: Graph folding of the clique-sum graph.

$\left.\left(u_{2}, u_{3}\right),\left(v_{1}, u_{2}\right),\left(v_{2}, u_{1}\right)\right\}=\left\{\left(v_{3}, v_{4}\right),\left(v_{3}, v_{4}\right), \quad\left(v_{4}, v_{3}\right),\left(u_{3}\right.\right.$, $\left.\left.u_{4}\right),\left(u_{3}, u_{4}\right),\left(u_{4}, u_{3}\right),\left(v_{3}, u_{4}\right),\left(v_{4}, u_{3}\right)\right\}$ is a graph folding, see Figure 9(b).

\section{Graph Folding of the Clique-Sum Graph}

We will denote the clique-sum of the two graphs $G$ and $H$ by $G$ cli $H$.

Definition 2. Let $G_{1}, G_{2}, G_{3}$, and $G_{4}$ be graphs. Let $f: G_{1} \longrightarrow G_{3}$ and $g: G_{2} \longrightarrow G_{4}$ be graph maps. Then, we can define a map from the clique-sum of $G_{1}$ and $G_{2}$ to the clique-sum of $G_{3}$ and $G_{4}$ denoted by $f$ cli $g$ : $G_{1}$ cli $G_{2} \longrightarrow G_{3}$ cli $G_{4}$ as follows:

\section{shared cliques.}

$$
(f \operatorname{cli} g)(e)=\left\{\begin{array}{l}
f(e), \quad e \in G_{1}, \\
f(e)=g(e)=e, \text { for all edges of the } \\
g(e), \quad e \in G_{2},
\end{array}\right.
$$

This map is called the clique-sum map of the maps $f$ and $g$

Theorem 8. Let $G_{1}, G_{2}, G_{3}$, and $G_{4}$ be graphs. Let $f: G_{1} \longrightarrow G_{3}$ and $g: G_{2} \longrightarrow G_{4}$ be graph foldings. Then, the clique-sum map fcli $g: G_{1} \mathrm{cli}_{2} \longrightarrow G_{3} \mathrm{cli}_{4}$ is a graph folding.

Proof. Suppose $f$ and $g$ are graph foldings. Now, let $e \in G_{1}$ cli $G_{2}$, and then either $e \in G_{1}$ or $e \in G_{2}$. In these two cases and since each of $f$ and $g$ is a graph folding, then $(f$ clig $)(e) \in\left(G_{3} \operatorname{cli} G_{4}\right)$. Thus, $(f$ cli $g)$ maps edges to edges, and hence the clique-sum map is a graph folding.

Example 7. Consider the two graphs $G_{1}$ and $G_{2}$ shown in Figure 10(a). Let $f: G_{1} \longrightarrow G_{1}$ be a graph folding defined by $f\left\{v_{5}\right\}=\left\{v_{4}\right\}, f\left\{e_{7}, e_{8}, e_{9}\right\}=\left\{e_{4}, e_{5}, e_{6}\right\}, g\left\{v_{6}, v_{7}, v_{8}\right\}=\left\{v_{1}, v_{2}\right.$, $\left.v_{3}\right\}$ and $g\left\{e_{17}, e_{18}, e_{12}, e_{16}, e_{10}, e_{11}, e_{13}, e_{14}, e_{15}\right\}=\left\{e_{1}, e_{3}, e_{3}\right.$, $\left.e_{1}, e_{3}, e_{2}, e_{2}, e_{1}, e_{2}\right\}$. Then, the clique-sum map $f$ cli $g$ : $G_{1}$ cli $_{2} \longrightarrow G_{1}$ cliG $_{2}$ defined by $(f$ cli $g)\left\{v_{5}, v_{6}, v_{7}, v_{8}\right\}=\left\{v_{4}\right.$, $\left.v_{1}, v_{2}, v_{3}\right\}$ and $(f \operatorname{cli} g)\left\{e_{7}, e_{8}, e_{9}, e_{17}, e_{18}, e_{12}, e_{16}, e_{10}, e_{11}, e_{13}\right.$, $\left.e_{14}, e_{15}\right\}=\left\{e_{4}, e_{5}, e_{6}, e_{1}, e_{3}, e_{3}, e_{1}, e_{3}, e_{2}, e_{2}, e_{1}, e_{2}\right\}$ is a graph folding, see Figure 10(b).

\section{Conclusion}

We obtained the necessary and sufficient conditions, if exist, for folding new graphs obtained from a given graph by some techniques like dual, gear, subdivision, web, crown, simplex, crossed prism, and clique-sum graphs. We can examine the relation between folding a given pair of graphs and folding of a new graph generated from these given pair of graphs by some operations like join, Cartesian product, normal product, and tensor product. Also, we can lift the definition of folding from graphs to digraphs which has close connections to important industrial applications.

\section{Data Availability}

No data were used to support this study. 


\section{Conflicts of Interest}

The authors declare that they have no conflicts of interest regarding the publication of this paper.

\section{References}

[1] R. Balakrishnan and K. Ranganathan, A Textbook of Graph Theory, Springer Science \& Business Media, Berlin, Germany, 2012.

[2] A. Brandstadt and J. P. Spinrad, Graph Classes: A Survey, vol. 3, SIAM, Philadelphia, PA, USA, 1999.

[3] R. J. Trudeau, Introduction to Graph Theory, Courier Corporation, Chelmsford, MA, USA, 2013.

[4] J. A. Gallian, "A dynamic survey of graph labeling," The Electronic Journal of Combinatorics, vol. 19, 2000.

[5] A. E. Brouwer and W. H. Haemers, "Distance-regular graphs," in Universitext, Spectra of Graphs Springer, New York, NY, USA, 2012.

[6] H.-J. Bandelt and M. Van De Vel, "Embedding topological median algebras in products of dendrons," Proceedings of the London Mathematical Society, vol. 58, no. 3, pp. 439-453, 1989.

[7] R. C. Read and R. J. Wilson, An Atlas of Graphs, Vol. 21, Clarendon Press, Oxford, UK, 1998.

[8] P. D. Seymour and R. W. Weaver, "A generalization of chordal graphs," Journal of Graph Theory, vol. 8, no. 2, pp. 241-251, 1984.

[9] P. Erdös, "Graph theory and probability," Canadian Journal of Mathematics, vol. 11, pp. 34-38, 1959.

[10] E. El-Kholy and A. El-Esawy, "Graph folding of some special graphs," Journal of Mathematics and Statistics, vol. 1, no. 1, pp. 66-70, 2005. 